\title{
Dental professionals' opinions and knowledge of smoking cessation and electronic cigarettes: a cross-sectional survey in the north of England.
}

Zuhaib Ahmed ${ }^{1}$, Philip M. Preshaw ${ }^{1}$, Linda Bauld ${ }^{2}$ and Richard Holliday ${ }^{1}$.

1. Centre for Oral Health Research, School of Dental Sciences, Newcastle University, Newcastle upon Tyne, UK

2. Institute for Social Marketing, Faculty of Health Sciences and Sport, University of Stirling, Stirling, UK

Authors have no conflict of interests.

Words count (3112)

'In brief' points:

- The majority of dental professionals in the north of England reported enquiring about their patients' smoking status and offering smoking cessation advice.

- The most important reported perceived barriers to providing smoking cessation advice were lack of time, lack of training and lack of patient interest. Half of dental professionals reported never receiving smoking cessation advice training.

- Dental professionals had very mixed views regarding e-cigarettes with approximately a third having negative views. Opinions had become increasingly negative in the year leading up to the survey, with one quarter of dental professionals reported that they recommended e-cigarettes to their patients who smoke. 


\section{Abstract}

Aims: To determine the current level of knowledge and opinions of UK dental professionals with regards to smoking cessation and e-cigarettes.

Methods: A self-administered online survey was distributed by postal invitation to all dental practices in the north of England registered on the National Health Service (NHS) Choices website.

Findings: One hundred and ninety completed questionnaires were received. Seventy-nine percent of respondents reported always enquiring about the smoking status of their patients with $17 \%$ completing referrals to a specialist stop smoking service. Just under half of respondents reported not receiving any smoking cessation advice training. Lack of time during appointments, lack of training and lack of perceived interest by patients were reported as the most important barriers. The importance of a lack of remuneration, as a barrier, varied considerably with professional role. Approximately a third (31\%) of respondents were of the opinion that e-cigarettes are more or equally harmful than cigarettes with the majority not aware of any guidance documents or recommendations regarding e-cigarettes.

Conclusion: The majority of dental professionals in the north of England reported providing smoking cessation advice, although only half had training on this. Opinions on electronic cigarettes were mixed, with a third having negative views. 


\section{Introduction}

Tobacco smoking remains the leading preventable cause of premature mortality and morbidity in the world ${ }^{1}$ and dental professionals have an important role to play in addressing smoking. Tobacco smoking has several detrimental effects on oral health including oral cancer, ${ }^{2}$ periodontitis, ${ }^{3}$ poor wound healing ${ }^{4}$ and tooth staining. ${ }^{5}$ Dental professionals are in an ideal position to offer smoking cessation advice (SCA) to a large proportion of the population. This is especially true in countries such as England and Wales where over three-quarters of dentate adults reported visiting the dentist at least once every 2 -years. ${ }^{6}$ There are also often tangible prompts that can be used when discussing the consequences of smoking with patients e.g. tooth staining, mobile teeth or tooth loss. In the United Kingdom (UK), guidance from the National Centre for Smoking Cessation and Training (NCSCT), Public Health England (PHE) and National Institute of Clinical Excellence (NICE) advises dentists to deliver a Very Brief Advice (VBA) intervention e.g. Three A's technique: Ask, Advise, Assist. ${ }^{7-9}$ A Cochrane review considered the effect of a brief advice intervention, delivered by oral health professionals in a range of dental settings (private, community, hospital and military). ${ }^{10}$ It reported a significant increase in the rate of smoking cessation, compared to "usual care, no contact or less treatment intensive controls." ${ }^{10}$ An odds ratio of 1.71 (95\% CI 1.44, 2.03) for tobacco abstinence was reported. ${ }^{10}$ A recent review of tobacco education in UK dental schools showed a generally positive situation indicating that new graduates should have received both theoretical and practical tobacco education. ${ }^{11}$

Our research group conducted a previous survey of dental professionals' attitudes and activities in relation to SCA across the north east of England in $2006 .{ }^{12}$ The survey reported that only a third of dental professionals reported 'always' enquiring about the 
smoking status of their patients with a third of dentists reporting that they did not offer SCA. Potential barriers to delivering smoking cessation advice included: lack of remuneration, lack of time and lack of training. A recent narrative review of tobacco cessation support in general dental practice included six cross-sectional studies published between 1997 and $2014 .{ }^{13}$ It reported that perceived time availability, and the smoking status of dental professionals, were important determinants of whether SCA was delivered. ${ }^{13}$

Since these studies were completed there have been several substantial changes to the field of smoking cessation including service restructuring, ${ }^{14}$ funding changes ${ }^{14}$ and the popularity of novel nicotine products such as electronic cigarettes (e-cigarettes). ${ }^{15}$ To date, there are no published surveys on the knowledge and opinions of dental professionals towards e-cigarettes. The north of England was chosen as the region of study for multiple reasons: it was the location of the previous survey, ${ }^{12}$ allowing comparison; it was geographically close to the researchers; and the north of England have some of the highest smoking rates in England. ${ }^{16}$ Therefore, the aim of this study was to investigate and evaluate the current knowledge and opinions of dental professionals in the north of England towards smoking cessation and e-cigarettes. 


\section{Materials and methods}

\section{Study cohort}

The study design was a self-administered online questionnaire survey of dental professionals (dentists, dental therapists and hygienists [DHTs], dental nurses). Ethical approval was obtained from the Newcastle University Research Ethics Committee (Reference number: 10345/2016). The NHS Choices website was used to identify all NHS dental practices within the North of England within the postcode regions: CA, DL, DH, TS, LA, SR, NE, and YO. A search completed on $17^{\text {th }}$ December 2016 identified 499 dental practices.

\section{Questionnaire}

The questionnaire consisted of 3 sections, with 21 research items in total (supplementary material 1). It included bivariate answers, multiple choice and Likert responses. The first section obtained demographic information about the respondents including: professional role, gender, age, graduation year and work locations and smoking status. The second section investigated provision of SCA including: frequency of enquiring about their patients' smoking status, importance of the dental team in providing SCA, personnel responsible for offering SCA, training in SCA, perceived barriers in delivering SCA and the most likely methods used for further stop smoking support. The third section investigated opinions and current practice regarding e-cigarettes and covered: perceived harmful effects of e-cigarettes compared to regular cigarettes, if e-cigarettes will be good for public health, changes in perception over the last 12 months, comfort in recommending e-cigarettes (including if a medically licensed product was available) and awareness of any guidance documents. 
Where possible, the questions in this survey were identical to those asked in previous studies $(2,9,11)$ to allow for comparison. The survey was conducted using online survey software (Qualtrics, Provo, Utah, US). The survey was configured so each question had to be answered in turn and it was not possible to return to a previous question. The survey was piloted with 15 dental professionals within the Newcastle Dental Hospital (Newcastle upon Tyne, UK) and refined following feedback.

\section{Data Collection procedure}

Postal invitations were sent to each dental practice, which included a covering letter (supplementary material 2) requesting that one member from each staff category (dentist, DHT, dental nurse) complete the survey. Business cards were included (one for each category) which had details of how to access the survey (web address and QR code). Participants had the option of entering into a raffle for vouchers if they completed the questionnaire ( $£ 100$ voucher for each staff category). A second postal invitation was sent after 30 days (where required).

\section{Data Analysis}

The data were cleaned and entered into Microsoft Excel 2013 (Microsoft Office Home and Business 2013, Version: 15.0.495.1001). Statistical Package for the Social Sciences (Windows version 23.0; SPSS Inc., Chicago [IL], US) was used to analyse data. Descriptive statistics were calculated for each variable. 


\section{Results}

\section{Demographic Details}

One hundred and ninety fully completed questionnaires were received, an overall response rate of $13 \%($ dentists $=16 \%[n=81]$, DHTs $=5 \%[n=24]$, dental nurses: $17 \%[n=85])$. The study population consisted of $76 \%(n=145)$ females, $23 \%(n=44)$ males and $1 \%(n=2)$ preferred not to provide their gender. Overall, $19 \%(\mathrm{n}=36)$ of respondents were working in multiple practices with this increasing to $63 \%(n=15)$ for DHTs. The graduation year of the participants ranged from 1970-2017. Demographic data are presented in Table 1.

\section{Smoking Status}

Self-reported tobacco smoking rates were low with just $6 \%(n=12)$ reporting being a current smoker and 24\% $(\mathrm{n}=46)$ reporting being a former smoker. Data regarding smoking status by professional role are shown in Table 1 .

\section{Smoking Cessation Advice}

The vast majority of dental professionals acknowledged the importance of the dental team in offering SCA. On a Likert scale of 0-5, with higher scores indicating increased importance, the median score was $5(\mathrm{IQR}=1)$. Seventy-nine percent $(\mathrm{n}=150)$ of dental professionals stated they 'always' enquired about the smoking status of their patients (19\% [n=36] 'sometimes', 2\% [n=4] 'never'). When asked about offering SCA, 77\% $(n=147)$ of dental professionals reported currently offering advice. With respect to their roles, more DHTs $(100 \%, \mathrm{n}=24)$ reported currently offering SCA compared to dentists $(81 \%, \mathrm{n}=66)$ and dental nurses $(67 \%, n=57)$. Of dental professionals who currently smoke, $58 \%(n=7)$ reported offered SCA compared to $79 \%(n=139)$ in non-smokers. With respect to their 
roles, more DHTs $(100 \%, \mathrm{n}=24)$ reported currently offering SCA compared to dentists $(81 \%, n=66)$ and dental nurses $(67 \%, n=57)$.

Respondents were asked which personnel within their practices should routinely offer SCA. The majority of the participants were of the view that the dentist $(96 \%, n=182)$ or DHT $(86 \%, n=164)$ should be responsible for routinely offering SCA. This decreased to $56 \%(\mathrm{n}=107)$ for dental nurses (Table 2).

For further stop smoking cessation support, $68 \%(\mathrm{n}=130)$ reported 'advising them to see their General Medical Practitioner (GMP)', 63\% $(n=119)$ reported 'advising them to contact/ attend a specialist stop smoking service', $33 \%(n=63)$ reported 'advising them to see their pharmacist' and $17 \%$ reported 'completing a referral to a specialist stop smoking service'. With the free text answer option $4 \%(n=7)$ reported using 'in-house' smoking cessation services.

When asked about previous training in smoking cessation, 26\% $(\mathrm{n}=49)$ reported receiving training during their dental qualification and 33\% $(n=62)$ reported receiving training since qualifying. Approximately half of participants $(51 \%, n=97)$ reported receiving SCA training at any time point (either pre- or post-qualification) (Table 3).

\section{Barriers}

Participants were asked to rate six potential barriers on a Likert scale of $0-5(0=$ not important at all, $5=$ very important). Lack of time during appointments, lack of training and lack of interest of patients all had a median value of 4. No involvements of colleagues, fear of damaging patient rapport and lack of remuneration all had a median value of 3 . The perceived barrier around lack of remuneration varied considerably with professional role. DHTs rated a lack of remuneration as not being an important barrier with $38 \%(\mathrm{n}=9)$ rating 
as 0 and $8 \%(\mathrm{n}=2)$ rating as 5 (median $=2, \mathrm{IQR}=3.75)$. Dentists rated a lack of remuneration as being an important barrier with $11 \%(\mathrm{n}=9)$ rating as 0 and $33 \%(\mathrm{n}=27)$ rating as 5 (median=4, IQR= 2.5) (Table 4).

\section{E-cigarettes}

Participants were asked to compare any harmful health effects of e-cigarettes relative to tobacco cigarettes. Approximately a third $(31 \%, \mathrm{n}=59)$ were of the opinion that e-cigarettes were more or equally harmful to tobacco cigarettes, $42 \%(n=79)$ less harmful, $15 \%(n=28)$ a lot less harmful and 13\% $(\mathrm{n}=24)$ reported not knowing (Figure 1).

Participants were asked to give an opinion on the statement, 'on balance, e-cigarettes will be good for the health of the public'. Thirty-five percent $(n=67)$ somewhat agreed or strongly agreed, $24 \%(n=45)$ neither agreed or disagreed and $41 \%(n=78)$ somewhat disagreed or strongly disagreed with the statement. Half of participants $(50 \%, \mathrm{n}=95)$ reported becoming less positive about e-cigarettes over the previous 12 months.

Participants were asked to give an opinion on the statement, 'I'm currently comfortable recommending e-cigarettes to my patients who smoke'. Twenty-seven percent $(n=51)$ somewhat agreed or strongly agreed, $21.5 \%(\mathrm{n}=41)$ neither agreed or disagreed and $51.5 \%$ ( $n=98)$ somewhat disagreed or strongly disagreed with the statement. There was little variation by professional role as detailed in Figure 2. A further similar question was asked but with respondents asked to consider their response under the circumstance that an ecigarette was available as a licenced medication. The participants responded more positively with $47 \%(n=90)$ somewhat agreeing or strongly agreeing, $28 \%(n=54)$ neither 
agreeing or disagreeing and $24 \%(n=46)$ somewhat disagreeing or strongly disagreeing (Figure 2).

The majority of dental professionals $(92 \%[n=175])$ were not aware of any guidance documents or recommendations regarding e-cigarettes. 


\section{Discussion}

In our survey, we contacted 499 practices from across the north of England (1497 potential participants) and achieved 190 responses, an overall response rate of $13 \%$. This response rate is low but is increasingly typical for surveys of health professionals operating in a busy environment with competing demands for time and where financial incentives for completion are not offered (this study offered entry into a prize draw). ${ }^{17-20} \mathrm{We}$ were limited to postal invitations due to the lack of email contacts. We were also unable to verify the accuracy of our sampling frame (NHS Choices) and it is possible some of the information was out-of-date, contributing to the low response rate. A recent study highlighted the challenges of obtaining high survey response rates from dental professionals and identified that these have been declining in recent years. ${ }^{21}$

The majority $(76 \%)$ of respondents were females with the gender distributions across the professions being very similar to those published in a recent workforce analysis within the region. ${ }^{22}$ It was also important to note that most of the dentists and dental nurses worked in single practices, unlike the DHTs, of whom $63 \%$ worked in multiple practices. The survey revealed that self-reported smoking rates were low (6\%), and were lower than those reported in our previous survey $(13 \%)^{12}$ and well below the UK national average $(15.8 \%){ }^{23}$

\section{Smoking Cessation}

It was encouraging to find that the vast majority of participants reported a positive attitude towards the role of dental team in smoking cessation. There was a substantial increase, compared to our previous survey, ${ }^{12}$ in the number of participants reporting always enquiring about the smoking status of their patients (79\% compared to $34 \%$ ). With regards to delivery of SCA, the vast majority reported delivering this to smokers (77\%) but it was noteworthy 
that still one-fifth of the dentists reported not offering SCA. Our results were in line with the findings of a recent narrative review which found that smoking among dental professionals was negatively associated with providing SCA. ${ }^{13}$

Our previous survey ${ }^{12}$ found that only $38 \%$ of participants expected dental nurses to offer SCA, with only $28 \%$ of dental nurses thinking they should offer advice. The current study showed a substantial shift in opinions in this regard, with $56 \%$ of participants having the opinion that dental nurses should offer SCA. Interestingly only $45 \%$ of dental nurses felt they should be routinely delivering SCA, although higher proportions of dentists (63\%) and DHTs $(71 \%)$ felt dental nurses should be doing this. A potential explanation for this increase is that in July 2008 it became compulsory for dental nurses in the UK to register with the General Dental Council (GDC) with mandatory continuing professional development (CPD) as a requirement of registration. This 'professionalization' of the role may have positively influenced the expectations of dental nurses (and others) towards their responsibilities. However, a survey in 2011 investigated the effect of GDC registration and found that most participants reported this had no effect on either their role as a dental nurse or status in the dental team. ${ }^{24}$ The results of our study may be beginning to show some longer-term impacts of the GDC registration process.

Our study identified a lack of time, training and interest from patients as the most important perceived barriers to providing SCA. This is in keeping with several other studies, for example, Ibrahim et al. ${ }^{25}$ found the same three barriers to be the most important in their sample of Malaysian dentists.

With regards to training, although this was perceived as an important barrier, it was less commonly reported than in our previous survey. ${ }^{12}$ Likewise, the proportion of participants 
who had received previous training on SCA had increased from $30 \%$ to $50 \%$. As in our previous survey, more DHTs had received training than in other groups (79\% compared to $45 \%$ in 2006). This may reflect the availability of training but also the expectations of individuals in the different professional roles $(100 \%$ of DHTs felt they should be offering SCA, compared to $95 \%$ of dentists and $45 \%$ of dental nurses).

A recent survey of tobacco education in UK dental schools ${ }^{11}$ showed a marked increase in the number of schools reporting providing tobacco education and assessment, compared to a survey in $2009 .{ }^{26}$ The results of the our study show a similar theme. Recent graduates ( $\geq 2010$ ) were twice as likely to report receiving SCA training during their dental qualification training compared to those who graduated prior to 2010 (Table 3). Our study also identified that only half of participants had received any previous SCA training, which highlights the need for further efforts in this field. The National Centre for Smoking Cessation and Training (NCSCT) have online accredited training, including a 'very brief advice training' module. ${ }^{7}$ However, this is targeted at GMPs and may be more appealing if a similar package was produced specifically for the dental team.

\section{E-cigarettes}

At present, there are 2.9 million users of e-cigarettes in the $\mathrm{UK},{ }^{27}$ almost all current smokers or former smokers. There is a growing consensus regarding e-cigarettes across public health and medical bodies in the UK that encourages their use by smokers as part of a comprehensive approach to tobacco control which includes tobacco harm reduction. ${ }^{28}$ Our study, the first we are aware of, tried to establish the views of the dental profession regarding e-cigarettes. Views of dental professionals were varied with over half of the opinion that ecigarettes were less or a lot less harmful for health compared to regular cigarettes. However, 
approximately a third of our respondents felt e-cigarettes were likely to be more or equally harmful for health compared to regular cigarettes, a view in contrast to that of the public health community and also interestingly more negative than that of the general public $(26 \%) .{ }^{27}$ However, there was considerable variation between professional roles with over half of dental nurses answering 'more or equally harmful' compared to under $15 \%$ of DHT and dentists. Unlike the general public, there were relatively few dental professionals who chose to answer 'don't know' and a relatively large portion answering 'less harmful' or a 'lot less harmful'. Another important finding was that opinions were generally becoming more negative towards e-cigarettes, which fits with the trends observed in the UK general population. ${ }^{27}$ Unsurprisingly, most practitioners were not currently comfortable recommending e-cigarettes to their patients who smoke, although the potential future availability of an e-cigarette as a licensed medication had a positive impact on this (Figures 2 and 3). Very few participants were aware of any guidance documents regarding ecigarettes, perhaps reflecting the lack of any from dental organisations. Examples of useful guidance documents include those by the $\operatorname{NCSCT}^{29}$ and Royal College of General Practitioners (RCGP). ${ }^{30}$ Tailored training and resources for dental professionals would help address the uncertainly and variation of opinion on e-cigarettes.

\section{Limitations}

The cross-sectional design of the study will be prone to selection bias (non-response and volunteer bias) meaning the sample is not representative of the population e.g. those who responded to the survey may have been more engaged individuals and more likely to have engaged with training and delivering SCA. ${ }^{31}$ The low response rate also means the results may not be representative of the population. Survey responses may also be subject to recall 
bias due to the self-reported nature of the data. The interpretation of the results should take these factors into account. 


\section{Conclusion}

The significant majority of dental professionals in a large region of England are enquiring about their patients' smoking status and offer SCA. The most important perceived barriers to this were lack of time, lack of training and lack of patient interest. The proportion of dental professionals who had received SCA training has increased compared to previous surveys but around half reported never receiving training. Dental professionals had very mixed views regarding e-cigarettes with over half having positive views and approximately a third having negative views. Opinions had become increasingly negative in the year leading up to the survey and only one quarter of dental health professionals reported that they recommended e-cigarettes to their patients who smoke. Very few participants were aware of any guidance documents regarding e-cigarettes. These findings suggest significant gaps exist amongst dental health professionals regarding e-cigarettes specifically, and for some, regarding smoking cessation in general. Tailored training and resources are required in the dental health setting to address this gap. 


\section{Declaration of Interests}

Richard Holliday is funded by a National Institute for Health Research Doctoral Research Fellowship (DRF-2015-08-077). This paper presents independent research funded by the National Institute for Health Research (NIHR). The views expressed are those of the authors and not necessarily those of the NHS, the NIHR or the Department of Health. None of the authors reports any conflict of interest. 


\section{Figures legends}

Figure 1. Views of dental professionals regarding the relative health harm of e-cigarettes compared to regular cigarettes by professional role. Data from the ASH/YouGov surveys (2013 and 2017) are shown for comparison. ${ }^{18}$

Figure 2. Views of dental professionals on recommending e-cigarettes by professional role.

Footnote: 1: 'I'm currently comfortable recommending e-cigarettes to my patients who smoke', 2: 'If an e-cigarette was a licensed medication, I would definitely recommend them to patients'. 


\section{References}

1. World Health Organisation. WHO Report on the Global Tobacco Epidemic, 2008: the MPOWER package. Geneva. 2008.

2. Hashibe M, Brennan P, Chuang S-c, et al. Interaction between tobacco and alcohol use and the risk of head and neck cancer: pooled analysis in the INHANCE consortium. Cancer Epidemiol Biomarkers Prev. 2009; 18: 541-550.

3. Tomar SL, Asma S. Smoking-attributable periodontitis in the United States: findings from NHANES III. National Health and Nutrition Examination Survey. $J$ Periodontol. 2000; 71: 743-751.

4. Palmer RM, Wilson RF, Hasan AS, Scott DA. Mechanisms of action of environmental factors--tobacco smoking. J Clin Periodontol. 2005; 32: 180-195.

5. Watts A, Addy M. Tooth discolouration and staining: a review of the literature. $\mathrm{Br}$ Dent J. 2001; 190: 309-316.

6. Steele J, O'Sullivan I. Executive Summary: Adult Dental Health Survey 2009. London: The Health and Social Care Information Centre. 2011.

7. National Centre for Smoking Cessation and Training. Very Brief Advice training module. Available from: http://www.ncsct.co.uk/publication_very-brief-advice.php. [accessed: October 2017].

8. NICE. Smoking: brief interventions and referrals (PH1). Manchester: National Institute for Health and Care Excellence, 2006.

9. Delivering better oral health: an evidence-based toolkit for prevention. Third Edition. London: Public Health England, 2014. 
10. Carr AB, Ebbert J. Interventions for tobacco cessation in the dental setting. The Cochrane database of systematic reviews 2012(6):Cd005084

11. Holliday R, Amin K, Lawrence V, Preshaw PM. Tobacco education in UK dental schools: A survey of current practice. Eur J Dent Educ 2017; Epub ahead of print.

12. Stacey F, Heasman PA, Heasman L, Hepburn S, McCracken GI, Preshaw PM. Smoking cessation as a dental intervention--views of the profession. Br Dent J 2006; 201:109-113.

13. Lala R, Csikar J, Douglas G, Muarry J. Factors that influence delivery of tobacco cessation support in general dental practice: a narrative review. J Public Health Dent 2017; 77: 47-53.

14. Feeling the Heat: The Decline of Stop Smoking Services in England. Findings from a survey of local authority tobacco control leads. London: Cancer Research UK, Action on Smoking and Health, 2018.

15. McNeill A, Brose L, Calder R, Bauld L, Robson D. Evidence review of e-cigarettes and heated tobacco products 2018. A report commissioned by Public Health England. London: Public Health England; 2018.

16. Statistics on Smoking. London: NHS Digital, 2017.

17. Hiscock R, Bauld L, Arnott D, Dockrell M, Ross L, McEwen A. Views from the Coalface: What Do English Stop Smoking Service Personnel Think about ECigarettes? Int J Environ Res Public Health 2015; 12: 16157-16167..

18. Blackwell E, Nesbit M, Petridis H. Survey on the use of CAD-CAM technology by UK and Irish dental technicians. Br Dent J 2017; 222: 689.

19. Tran D, Nesbit M, Petridis H. Survey of UK dentists regarding the use of CAD/CAM technology. Br Dent J 2016; 221: 639. 
20. Kalsi AS, Kochhar S, Lewis NJ, Hemmings KW. New UK graduates' knowledge of training and service provision within restorative dentistry - a survey. Br Dent J 2017; 222: 881 .

21. Funkhouser E, Vellala K, Baltuck C, et al. Survey methods to optimize response rate in the national dental practice-based research network. Eval Health Prof 2017; 40: $332-358$.

22. Vance G HR, Burford B, Shapiro E. Planning and developing the dental workforce of the future. A survey of the dental workforce in North East England and North Cumbria. Newcastle upon Tyne: Newcastle University, 2016. Available at: https://madeinheene.hee.nhs.uk/Portals/13/Dental\%20Workforce\%20Survey\%20final. pdf. [accessed October 2017].

23. Office of National Statistics. Statistical bulletin: Adult smoking habits in the UK: 2016. London: Office of National Statistics, 2017

24. Turner S, Ross M, Ibbetson R. The impact of registration and CPD on dental nurses. Vital 2012; 9: 24-31.

25. Ibrahim H NS. Attitudes and practices in smoking cessation counselling among dentists in Kelantan. Arch Orofac Sci 2008; 3: 11-16.

26. Ramseier CA, Aurich P, Bottini C, Warnakulasuriya S, Davis JM. Curriculum survey on tobacco education in European dental schools. Br Dent J 2012; 213: E12

27. ASH Fact Sheet. Use of e-cigarettes (vapourisers) among adults in Great Britain. London: Action on Smoking and Health (ASH), 2017.

28. E-cigarettes: a developing public health consensus. Joint statement on e-cigarettes by Public Health England and other UK public health organisation. PHE publications gateway number: 2016129. London: Public Health England, 2016. 
29. Electronic cigarettes: A briefing for stop smoking services. London: National Centre for Smoking Cessation and Training, 2016. Available at:

http://www.ncsct.co.uk/publication_electronic_cigarette_briefing.php [Accessed Janaury 2018].

30. RCGP Position Statement on the use if electronic nicotine vapour products (Ecigarettes). London: Royal College of General Practitioners, 2017. Available at: http://www.rcgp.org.uk/policy/rcgp-policy-areas/e-cigarettes-non-combustibleinhaled-tobacco-products.aspx [Accessed January 2018].

31. Sedgwick P. Bias in observational study designs: cross sectional studies. BMJ 2015; 350: h1286. 